\title{
Challenges of Improving Intensive Care Medicine in Eritrea: Impact of an Italian Cooperative Project of Educational and Clinical Support ${ }^{*}$
}

\author{
Valentina Anichini ${ }^{1}$, Giovanni Zagli ${ }^{1 \#}$, Hagos Goitom ${ }^{2}$, Giovanni Cianchi ${ }^{1}$, Andrea Cecchi ${ }^{1}$, \\ Lucia Perretta $^{1}$, Emanuele Bigazzi ${ }^{1}$, Barbara Gazzini ${ }^{1}$, Simone Proietti ${ }^{1}$, Alessandro Di Filippo ${ }^{3}$, \\ Simone Toccafondi ${ }^{4}$, Gianfranco Gensini ${ }^{5}$, Giancarlo Berni ${ }^{6}$, Adriano Peris ${ }^{1}$
}

\begin{abstract}
${ }^{1}$ Anesthesia and Intensive Care Unit of Emergency Department, Careggi Teaching Hospital, Florence, Italy; ${ }^{2}$ Department Intensive Care Unit, Orotta National Referral Hospital, Asmara, Eritrea; ${ }^{3}$ Anesthesia and Intensive Care, Faculty of Medicine, University of Florence, Florence, Italy; ${ }^{4}$ First Aid of Emergency Department, Careggi Teaching Hospital, Florence, Italy; ${ }^{5}$ Department of Critical Care Medicine and Surgery, Careggi Teaching Hospital, Florence, Italy; ${ }^{6}$ Tuscany Region Sanitary System, Florence, Italy.

Email: " giovanni.zagli@unifi.it
\end{abstract}

Received June $8^{\text {th }}, 2013$; revised July $8^{\text {th }}, 2013$; accepted July $31^{\text {st }}, 2013$

Copyright (C) 2013 Valentina Anichini et al. This is an open access article distributed under the Creative Commons Attribution License, which permits unrestricted use, distribution, and reproduction in any medium, provided the original work is properly cited.

\begin{abstract}
Intensive care in Africa is available only in teaching or referral hospitals. Here we report the experience of a multidisciplinary collaboration between physicians and nurses of the Emergency Department (First Aid and Intensive Care Unit) of a tertiary referral hospital (Careggi Teaching Hospital, Florence, IT) and physicians and nurses of Orotta National referral Hospital in Asmara, Eritrea. The project was aimed at performing clinical assistance and training on the job to the local staff to improve the standard of care in the local Emergency Department. The duration of the project was initially planned to be 30 months, but unfortunately it was interrupted after 18 months because of lack of funds. The Italian staff was composed of two physicians and two nurses per period. To monitor local ICU activity, a retrospective survey of 36 months was performed. During the 36 months of data collection, 1169 patients were admitted to the ICU. Intra-ICU mortality rate resulted comparable before, during, and after Italian presence. On the contrary, the 28-day mortality resulted significantly lower bo th during and after the Italian stay. After project interruption, the Italian staff maintained contact with the Eritrean ICU personnel, who were invited to attend the Italian ICU for one month per year, and collected information about Orotta ICU activities.
\end{abstract}

Keywords: Intensive Care Unit; Developing Countries; MEWS

\section{Introduction}

Intensive care in Eritrea is available only in teaching or referral hospitals, even if in developing countries acute critical illness is often potentially reversible with adequate intensive care treatment. Expensive technologies, maintenance inadequacy, and shortage of health personnel can explain the difficulties in having a high level standard of care.

The Eritrean State has been independent since 1993. The population is about 5 million. Inhabitants have a life expectancy of 61 - 65 years (male-female), and the infant mortality rate is $7.4 \%$. Gross national income per capita

\footnotetext{
*Conflicts of Interest: The authors declare that they do not have any conflicts of interest.

\#Corresponding author.
}

is $\$ 680$, and total expenditure on health per capita is $\$ 28$. (WHO statistics:

http://www.afro.who.int/en/eritrea/country-health-profile .html). Information on the Eritrean population health status is available on the world health statistics site (http://www.who.int/whosis/en/). Another important paper describing the health status of the Eritrean population reports the prevalence of non-communicable disease risk factors [1]. Here we report the experience of a multidisciplinary collaboration among physicians and nurses of the Emergency Department (First Aid and Intensive Care Unit) of a tertiary referral hospital (Careggi Teaching Hospital, Florence, IT). The collaboration project, sustained by the Eritrean Health Ministry and the Tuscan Region, took place in the Orotta National referral Hospi- 
tal in Asmara, Eritrea.

In a recent review, critical care improvement as a fundamental aspect of healthcare in the developing world has been emphasised [2]. In their article, Riviello and co-authors correctly underlined that the intensive care setting must be separate from the concept of "expensive" intensive care; instead, it must be thought in terms of "appropriate" intensive care, thus strictly related to the site of intervention. In this perspective, the introduction of the routine use of a simple and standardised score, such as Modified Early Warning System (MEWS) proposed by Subbe and co-workers [3,4], represents an example of intensification of standard of care without any significant impact on economic resources. This simple but important process can be realised thanks both to the existing ICU reality and the favourable and constructive collaboration between Eritrean and Italian physicians.

The goal of the project was to introduce a triage system in the emergency room, and treatment protocols in the operating theatre and in the Intensive Care Unit, according to international guidelines.

The project included many activities:

- Italian staff performed clinical assistance on Orotta patients;

- Teaching support to Eritrean staff was organised with frontal classes twice a week, as well as simulation courses. Moreover, on-the-job training activities were performed during the daily working hours of the nurses and doctors, with skills and knowledge assessed by Italian tutors;

- Supply of medical equipment, such as a blood gas analyser, an ultrasound machine, a ventilator machine, sedation drugs, and thrombolytic drugs.

At the end of the project, Eritrean personnel were thought to be able to manage critical medical or surgical scenarios and to transfer skills to nurse and medical students.

Italian staff permanence was 18 months. The entire project cost of 1,050,000 euros was paid by the Tuscan Region, and was used for human resources (insurance, salaries, travel, and lodging expenses), medical equipment, and shipping charges. After Italian departure, collaboration continued for 3 years more, offering 1 month attendance periods to the Eritrean staff in the Careggi Hospital ICU, to share the experience of intensive care with proper clinical assessment, treatment protocols, and bedside procedures on critical patients. Information about Orotta ICU activities after Italian departure was collected by Eritrean staff.

\section{Setting and Methods}

The co-operation project was aimed at performing clinical assistance and training to the local staff to improve the standard of care in the local Emergency Department. The effective duration of the project was 3 years (20052007), including 6 months for needs analysis and planning of intervention. The Italian staff was composed of two physicians and two nurses per period (usually two months each), working in the emergency room, the ICU, and the operating theatre. Orotta Hospital in Asmara was selected in the Eritrean Health Sheet as the referral hospital to treat medical and surgical emergencies, because critical patients could not receive proper care in peripheral health centres.

Orotta Hospital was built with a contribution from the Chinese government in 2003. It includes 300 beds, with surgical and medical wards, and an outpatient department for medication, laboratory examination, and diagnostic procedures. A central laboratory with blood units and a radiology department are available, with an ultrasound device, and a CT-scan. Only traditional X-rays are available bedside.

The Emergency Department includes 4 operating rooms, the emergency room, and 9 ICU beds, all supplied with centralised oxygen. Surgical activity in the Orotta is well organised, with numerous scheduled interventions, 3 surgeons, some medical students, and many anaesthesia nurses. A recovery room is available to care for patients after surgery, before returning to the ward.

The ICU is equipped with 3 multi-parametric monitors and 2 ventilators. The ICU staff is composed of 4 nurses, 8 nurse assistants, and one doctor in the morning with internist competencies. Consultants on call are available to treat obstetric, paediatric, neurosurgical, and urological patients. Another doctor is in charge of the emergency room during the day, working with 6 nurses and 6 nurse assistants. One of the nurses is responsible for the triage system, and the emergency room is equipped with a defibrillator, a multi parametric monitor, and some advanced airway devices.

During the night shift, only one physician was on duty at the hospital, and he decided whether to admit patients to the medicine or surgical ward, to the ICU, or to discharge them.

As simple evaluating tool for early identification of medical patients who need intensive care, the MEWS score was introduced in Orotta Hospital. The MEWS is a tool for bedside evaluation based on five physiological parameters: systolic blood pressure, pulse rate, respiratory rate, urinary output, body temperature, and neurological status (Table 1). A total score of 4 or more was considered as a ward alert. A MEWS score was retrospectively collected in all ICU patients, because all five parameters were registered on ICU admission clinical papers. The MEWS score was also taught during frontal lessons, in order to train nurses to call for the doctor on duty when a patient began to worsen, in a timely manner. 
Table 1. The modified early warning score (MEWS).

\begin{tabular}{|c|c|c|c|c|c|c|c|}
\hline $\begin{array}{l}\text { Systolic blood pressure } \\
(\mathrm{mmHg})\end{array}$ & $<70$ & $71-80$ & $81-100$ & $101-199$ & & $\geq 200$ & \\
\hline Heart rate per min & & $<40$ & $41-50$ & $51-100$ & $101-110$ & $111-129$ & $\geq 130$ \\
\hline Respiratory rate per min & & $<9$ & & $9-14$ & $15-20$ & $21-29$ & $\geq 30$ \\
\hline Temperature $\left({ }^{\circ} \mathrm{C}\right)$ & & $<35$ & & $35.1-38.4$ & & $\geq 38.5$ & \\
\hline Neurological status & & & & Alert & Reacting to voice & Reacting to pain & Unresponsive \\
\hline Scores & 3 & 2 & 1 & $\mathbf{0}$ & 1 & 2 & 3 \\
\hline
\end{tabular}

To monitor the Orotta ICU activity and to improve cooperation effectiveness, a retrospective survey of 36 months (from 6 months before Italian stay to 12 months after Italian stay) was done, including the following data: age, sex, admission cause, length of stay, and mortality in ICU. No seriousness of illness score was used, due to the lack of laboratory data.

GraphPad Prism 5 (GraphPad Software Inc., San Diego, CA) was used for statistical analysis. Continuous variables were analysed with Kruskal-Wallis test, whereas categorical data were examined using Chi-square test and Fisher's exact text (95\% confidence interval). P values were considered significant if less than 0.05 .

\section{Results}

During the 36 months of data collection, a total of 1169 patients were admitted in ICU. Table 2 summarizes demographic and clinical characteristics of patients of the 3 periods of data collection. During the Italian stay, admission diagnosis was represented mostly by medical disease $(85 \%)$, with only $8 \%$ of patients coming from the operating theatre, and $7 \%$ represented by trauma patients. Regarding medical patients, cardiovascular problems are widespread: $21 \%$ acute coronary syndrome, $9 \%$ deep venous thrombosis, $8 \%$ rheumatic heart failure. Other medical admissions are represented by respiratory problems (15\% pneumonia), and HIV and tuberculosis infections are frequent comorbidities.

During the Italian stay, ICU admission resulted to be higher than during the other periods. Intra-ICU mortality rate resulted comparable before, during, and after Italian presence. Due to data missing, the 28-days mortality could not be used as an outcome parameter, even if a decreasing trend was observed by the operator during the Italian stay and the later period. During the same two periods, MEWS scoring resulted comparable, and the mean ICU length of stay higher, than the retrospective control (Table 2).

Data collection from the Orotta ICU is still going on, and we report data about ICU mortality and length of stay related to the three following years (Table 3). No
Table 2. Baseline and outcome data of patients retrospectively collected (Jan 2005-June 2006), during the Italian experience and MEWS introduction (July 2006-Dec 2007), and after Italian experience (Jan 2008-Dec 2008).

\begin{tabular}{|c|c|c|c|}
\hline & $\begin{array}{c}\text { January } \\
2005 \\
\text { June } 2006\end{array}$ & $\begin{array}{c}\text { July } 2006 \\
\text { December } \\
2007\end{array}$ & $\begin{array}{c}\text { January } 2008 \\
\text { December } \\
2008\end{array}$ \\
\hline N patients & 294 & 515 & 349 \\
\hline Age, years (mean) & 48.4 & 48.8 & 45.2 \\
\hline Female sex (\%) & $46 \%(135)$ & $45 \%(232)$ & $47 \%(169)$ \\
\hline \multicolumn{4}{|l|}{ Admission (\%) } \\
\hline Medical & $87 \%(256)$ & $86 \%(443)$ & $83 \%(299)$ \\
\hline Surgical & $7 \%(21)$ & $7 \%(36)$ & $9 \%(32)$ \\
\hline Trauma & $6 \%(18)$ & $7 \%(36)$ & $5 \%(18)$ \\
\hline MEWS (mean) & 4.5 & 5.4 & 5.1 \\
\hline $\begin{array}{l}\text { ICU LOS, days } \\
\text { (mean) }\end{array}$ & 6.5 & $8.3^{* *}$ & $7.9^{* *}$ \\
\hline $\begin{array}{c}\text { ICU mortality, \% } \\
(\mathrm{N})\end{array}$ & $33 \%(97)$ & $35 \%(180)$ & $28 \%(101)$ \\
\hline
\end{tabular}

Table 3. Data collection of Orotta ICU data of activities during 3 years following project interruption.

\begin{tabular}{cccc}
\hline & $\mathbf{2 0 0 9}$ & $\mathbf{2 0 1 0}$ & $\mathbf{2 0 1 1}$ \\
\hline Number of ICU patients & 283 & 326 & 299 \\
ICU mortality (\%) & $36.7 \%$ & $39.8 \%$ & $42.8 \%$ \\
ICU LOS, days (mean) & 6.8 & 4.4 & 6.2 \\
\hline
\end{tabular}

information is available about severity of illness of ICU admitted patients. In the year 2009 part of the ICU staff was moved to a new dialysis unit, renewing about $2 / 3$ of the nurses trained before.

\section{Discussion}

The use of MEWS as a critical illness indicator permitted 
us to identify a low risk group (MEWS $<5$ ) and a high risk group (MEWS $>5$ ), typically with severe organ dysfunction. The usefulness of such a scoring system is its capability to quickly detect patients that need more accurate assessment and targeted intensive care treatment. The use of scoring systems to assess severity of illness of admitted patients is a common practice in all developed countries. The impact of such analysis could be stronger in limited resource countries, where the burden of expensive critical care is relevant both to the hospital and to the patient. These scoring systems include physiological and laboratory indicators which usually are not available in African settings, thus, comparing the quality of care in different countries, or care before and after intervention, becomes a difficult task, given the difficulty in standardising severity of illness [5]. As in many other Track and Trigger warning systems, the introduction of MEWS was aimed at quickly recognising patients with potential or established critical illnesses, and to assure appropriate care by skilled staff in a proper setting (ICU). In the Orotta ICU, MEWS was introduced as a clinical assessment method, easily usable by the nursing staff. No trigger threshold was established, but parameters detected in the MEWS were part of alarm criteria, according to which, those who performed the clinical assessment would call for the doctor in charge. In the period after the Italian stay, although the number of patients decreased, clinical assessment of ICU admitted patients resulted similar (Table 2). After Italian departure, unfortunately no MEWS assessment was done. We don't know if the ICU mortality increase observed from 2008 to 2011 could be related to more critical patients or, more probably, to the change of staff.

Patients observed in the Orotta emergency department during our experience had mostly cardiovascular and respiratory diseases (in essence medical patients). Concerning cardiovascular disease, we observed a high incidence of acute coronary syndromes and heart failure. The first is epidemiological compared to occidental data, and may reflect improvement in lifestyle in the capital city. Heart failure was often observed in young patients presenting cardiac valve disease from rheumatic aetiology, living in rural areas with difficult access to health services. Respiratory diseases, as in many African countries, reflect tuberculosis or HIV prevalence. The increase in ICU admission during the Italian stay may be related to the strong working relationship between the emergency room and the ICU, and the efficacy of the triage programme performed in the emergency room.

Our results are limited by the lack of a follow-up program aimed at monitoring the long term maintenance of improvement obtained: this can be easily attributed to limited resources both in the local area and in the inter- national program. A strong limitation to this data collection is the missing data regarding 28-days survival after ICU discharge. The major problem was that we had survival information only if patients were still in the hospital. In many cases, no information about patients discharged to home were available, neither if they were dead nor alive.

\section{Conclusion}

As reported by attendant Eritrean people in the Italian ICU, the Orotta staff introduced some bed-side procedures in daily practice (ultrasound scan, tracheotomy, antimicrobial policy, patient positioning) [6,7], thus improving the quality of care in the hospital. Nevertheless, adequate effectiveness indicators are necessary to properly conduct a co-operation project, and time to teach "how to train" new staff must be prolonged over the years with the local presence of trainers and a response system assessment.

\section{Acknowledgements}

This project was supported by institutional funds (Tuscan Region and Careggi Teaching Hospital). The authors have reported that no significant conflicts of interest exist with any companies/organizations whose products or services may be discussed in this article.

A special thanks to the Sanitarians of Orotta Hospital.

\section{REFERENCES}

[1] A. Usman, G. Mebrahtu, J. Mufunda, P. Nyarango, H. Goitom, A. Kosia, Y. Ghebrat, A. Mosazghi, S. J. Atanga and M. M. Equbamichael, "Prevalence of Non-Communicable Disease Risk Factors in Eritrea," Ethnicity \& Disease, Vol. 16, No. 2, 2006, pp. 542-546.

[2] E. D. Riviello, S. Letchford, L. Achieng and M. W. Newton, "Critical Care in Resource-Poor Settings: Lessons Learned and Future Directions," Critical Care Medicine, Vol. 39, No. 4, 2011, pp. 860-867. doi:10.1097/CCM.0b013e318206d6d5

[3] C. P. Subbe, M. Kruger, P. Rutherford and L. Gemmel, "Validation of a Modified Early Warning Score in Medical Admissions," QJM: An International Journal of Medicine, Vol. 94, No. 10, 2001, pp. 521-526.

[4] C. P. Subbe, R. G. Davies, E. Williams, P. Rutherford and L. Gemmell, "Effect of Introducing the Modified Early Warning Score on Clinical Outcomes, Cardio-Pulmonary Arrests and Intensive Care Utilisation in Acute Medical Admissions," Anaesthesia, Vol. 58, No. 8, 2003, pp. 797 802. doi:10.1046/j.1365-2044.2003.03258.x

[5] R. M. Towey and S. Ojara, "Intensive Care in the Developing World," Anaesthesia, Vol. 62, No. Suppl 1, 2007, pp. 32-37. doi:10.1111/j.1365-2044.2007.05295.x

[6] H. Goitom and S. Dania, "Case Report Percutaneous 

of Educational and Clinical Support

Dilatational Tracheostomy: A Bedside Procedure in the Intensive Care Unit," Journal of Eritrean Medical Association, Vol. 3, No. 1, 2008, pp. 45-46.

[7] H. Goitom, "Lower Extremity Deep Vein Thrombosis among Intensive Care Patients in Orotta National Referral Hospital, Asmara, Eritrea," Journal of Eritrean Medical Association, Vol. 4, No. 1, 2009, pp. 1-4. 